\title{
Impact of macrolide therapy on mortality for patients with severe sepsis due to pneumonia
}

\author{
M.I. Restrepo*,\#, , E.M. Mortensen ${ }^{*,{ }^{\top},+}$, G.W. Waterer ${ }^{\S}$, R.G. Wunderink ${ }^{f}$, \\ J.J. Coalson** and A. Anzueto ${ }^{\#, \oplus}$
}

ABSTRACT: Recent studies suggest that macrolides may have beneficial effects for patients at risk for certain infections. The current authors examined the effect of macrolide therapy on 30 and 90-day mortality for patients with severe sepsis caused by pneumonia.

A retrospective cohort study was conducted at two tertiary teaching hospitals. Eligible subjects were admitted with a diagnosis of, had chest radiography consistent with, and had a discharge diagnosis of pneumonia and clinical criteria of severe sepsis. Subjects were considered to be on macrolides if they received at least one dose within $48 \mathrm{~h}$ of admission.

Severe sepsis was present in 237 (30.1\%) subjects, out of whom 104 (43.9\%) received macrolides. Mortality was $20.3 \%$ at 30 days and $24.5 \%$ at 90 days. In the multivariable analysis, the use of macrolide was associated with decreased mortality at 30 days (hazard ratio (HR) 0.3 , 95\% confidence interval $(\mathrm{Cl}) 0.2-0.7$ ) and at 90 days ( $\mathrm{HR} 0.3,95 \% \mathrm{Cl} 0.2-0.6$ ) in patients with severe sepsis and in patients with macrolide-resistant pathogens (HR 0.1, 95\% $\mathrm{Cl} 0.02-0.5$ ).

Macrolide use was associated with decreased mortality in patients with severe sepsis due to pneumonia and macrolide-resistant pathogens. Confirmatory studies are needed to determine whether macrolide therapy may be protective for patients with sepsis.

KEYWORDS: Antibiotics, community-acquired pneumonia, outcomes, sepsis

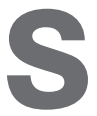
epsis affects $>700,000$ patients annually in the USA [1]. In addition, severe sepsis is the ninth leading cause of death in the USA and is associated with a high fatality rate, significant morbidity and great financial cost [2]. Respiratory infections, whether community- or hospital-acquired, account for the largest number of sepsis cases [1-3]. Community-acquired pneumonia (CAP) is one of the most common reasons for sepsis and is itself, independent of sepsis, the seventh leading cause of death and the leading cause of infectious death in the USA [4]. Although mortality due to pneumonia decreased significantly with the introduction of antibiotics in the 1950s, since that time mortality has been stable or increasing [5]. Mortality due to severe sepsis remains unacceptably high despite aggressive resuscitation and organ support, combined with appropriate antibiotics [6] and new therapies such as recombinant human activated protein C [3].

Recent studies suggest that macrolides may have beneficial effects for patients at risk from certain infections, due to their immunomodulatory effects rather than their antimicrobial properties [7]. These immunomodulatory effects are supported by the clinical success observed in noninfectious, but immune-related, conditions, including asthma [8], chronic obstructive pulmonary disease (COPD) [9], diffuse panbronchiolitis [10] and bronchiectasis [10]. In addition, much of the beneficial effect of macrolides seen in patients with bacteraemic pneumococcal pneumonia [1116] and CAP may also be due to the immunomodulatory effects rather than solely the antimicrobial effects [17-20]. Cytokines play an important role in host defence mechanisms for patients with CAP and severe sepsis [21]. Several cytokines, including tumour necrosis factor- $\alpha$, interleukin (IL)-1, IL-6 and IL-10 have been associated with sepsis [21]. Macrolides have been demonstrated to modulate these cytokines, which is potentially the basis for their beneficial effect [22].

The aim of the present study was to assess the effect of macrolide therapy on short- (30 days)
AFFILIATIONS

*Veterans Evidence-Based Research Dissemination Implementation Center Audie L. Murphy Veterans Affairs Hospital, \#Division of Pulmonary and Critical Care Medicine,

'South Texas Veterans Health Care System Audie L. Murphy Division, ${ }^{+}$Depts of Medicine, and

**Pathology, Division of General Medicine University of Texas Health Science Center at San Antonio, San Antonio, TX, and ${ }^{f}$ Northwestern University Feinberg School of Medicine, Chicago, IL, USA.

${ }^{\S}$ School of Medicine and Pharmacology Royal Perth Hospital Unit, University of Western Australia, Perth, Australia.

\section{CORRESPONDENCE}

M.I. Restrepo, VERDICT (11C6), South Texas Veterans Health Care System Audie L. Murphy Division, 7400 Merton Minter Boulevard, San Antonio, TX 78229, USA. Fax: 12105674423

E-mail: restrepom@uthscsa.edu

Received:

April 082008

Accepted after revision:

August 122008

\section{SUPPORT STATEMENT}

M.I. Restrepo is supported by a Department of Veterans Affairs

(Washington, DC, USA) Veterans Integrated Service Network 17 new faculty grant and a National Institutes of Health (Bethesda, MD, USA) KL2 Grant. E.M. Mortensen was supported by Howard Hughes Medical Institute (Chevy Chase, MD, USA) facultystart up grant 00378-001 and a Department of Veterans Affairs Veterans Integrated Service Network 17 new faculty grant. The views expressed in the present article are those of the authors and do not necessarily represent the views of the Department of Veterans Affairs. 
and long- (90 days) term mortality for patients with severe sepsis caused by CAP, and in patients with documented macrolide-resistant pathogens.

\section{METHODS}

The present study was a retrospective cohort study of hospitalised CAP patients at two academic teaching tertiary care hospitals in San Antonio (TX, USA). The Institutional Review Board of the University of Texas Health Science Center (San Antonio) evaluated the research protocol and gave it exempt status.

\section{Study sites/inclusion and exclusion criteria}

All patients admitted to the study hospitals between January 1, 1999 and December 31, 2002 with a primary discharge diagnosis of pneumonia (International Classification of Diseases (ICD)-9 codes 480.0-483.99 or 485-487.0) or secondary discharge diagnosis of pneumonia with a primary diagnosis of respiratory failure (518.81) or sepsis (038.xx) were identified. Inclusion criteria were: 1 ) age $\geqslant 18$ yrs; 2 ) admission diagnosis of CAP; and 3) abnormal chest radiography or chest computed tomography within $24 \mathrm{~h}$ of admission consistent with CAP. To be included, patients also had to have met the definition of severe sepsis based on the evidence of infection (CAP) with the presence of at least one organ dysfunction as recommended by the American College of Chest Physicians consensus definition [23].

Exclusion criteria included: 1) discharge from an acute care facility within 14 days of admission; 2) admission to another acute care hospital or nursing home; 3) HIV/AIDS; and 4) having "comfort measures only" status during the admission. If a subject was admitted more than once during the study period, only the first hospitalisation was abstracted.

\section{Clinical outcomes}

The primary outcomes were 30- and 90-day mortality and the secondary outcome was length of hospital stay. Both 30- and 90-day mortality were included in order to differentiate between the proportion of deaths attributable to pneumonia (30-day) and to other causes (90-day) [24]. Mortality was assessed using information from the Texas Dept of Health (Austin, TX, USA) and Dept of Veterans Affairs (Washington, DC, USA) clinical database.

\section{Data abstraction}

Chart review data included: demographics, comorbid conditions, physical examination findings, laboratory data, guideline-concordant empirical antibiotic therapy, and chest radiographic reports. Comorbid conditions were identified from either the admission or discharge notes or outpatient problem lists. Antimicrobial therapy was considered guideline-concordant if it agreed with the 2007 Infectious Diseases Society of America/American Thoracic Society guidelines [25]. Information on macrolides (including erythromycin, clarithromycin and azithromycin) given within the first $48 \mathrm{~h}$ of admission was obtained.

\section{Diagnostic criteria}

Microbiological data results were reviewed, and a microbiological cause was assigned independently by two of the current investigators (M.I. Restrepo and E.M. Mortensen). The cause of pneumonia was stratified as definitive or presumptive. The definitive diagnosis was considered if one or more of the following conditions were met: 1) positive blood cultures for bacterial or fungal pathogens (in the absence of an extrapulmonary source of infection); 2) pleural fluid cultures yielding a bacterial pathogen; 3) endotracheal aspirates with moderate or heavy growth of bacterial pathogens; 4) significant quantitative culture growth from bronchoscopic respiratory samples (protected specimen brush cultures of $\geqslant 10^{3}$ colony forming units $(\mathrm{cfu}) \cdot \mathrm{mL}^{-1}$ and in bronchoalveolar lavage of $\left.\geqslant 10^{4} \mathrm{cfu} \cdot \mathrm{mL}^{-1}\right)$. A presumptive diagnosis was made if a qualitative valid sputum sample yielded one or more predominant bacterial pathogens. Definitive and presumptive causes were combined for reporting purposes. When two or more microbiological causes were present, the patient was considered to have a polymicrobial infection. A patient was considered to have CAP of unknown cause if microbiological studies were not performed or were inconclusive. In vitro susceptibilities were documented according to the National Committee for Clinical Laboratory Standards [26]. The classification of macrolide-resistant pathogens was made using the addition of naturally resistant pathogens, such as the Gram-negative rods (including Pseudomonas aeruginosa, Acinetobacter spp., Escherichia coli, Klebsiella pneumoniae and Proteus mirabilis, etc.) and those Gram-positive cocci (including macrolide- or penicillin-resistant Streptococcus spp. and methicillin-resistant Staphylococcus aureus) with documented resistance based on guidelines [25]. Documented Haemophilus spp. were excluded from the analysis due to the lack of macrolide susceptibility documentation.

\section{Statistical analyses}

Univariate statistics were used to test the association of demographic and clinical characteristics with all-cause 30and 90-day mortality. Categorical variables were analysed using the Chi-squared test and continuous variables were analysed using an unpaired t-test.

A propensity score technique was used to balance covariates associated with macrolide use between groups [27]. The use of the propensity score technique provides a way, in nonrandomised studies, to control for pre-treatment differences by defining sets of comparable patients. The propensity score was derived from a logistic regression model. A dichotomous indicator variable indexing whether a patient was on a macrolide was the response variable. The covariates used in the propensity score model were the pneumonia severity index (PSI) score (which includes comorbid conditions such as congestive heart failure, liver disease, renal disease, cerebrovascular disease and history of stroke) [28], COPD, admission to the intensive care unit (ICU) and guideline-concordant antibiotic therapy. An ordered categorical variable was created, based on a quartile stratification of the propensity score, to include in the Cox and regression models.

Multivariable analysis was performed using a Cox proportional hazards model with either 30- or 90-day mortality as the dependent variable [24, 28]. Survival curves were based on Cox proportional hazards modelling. Subgroup analyses were performed according to the susceptibility patterns. 


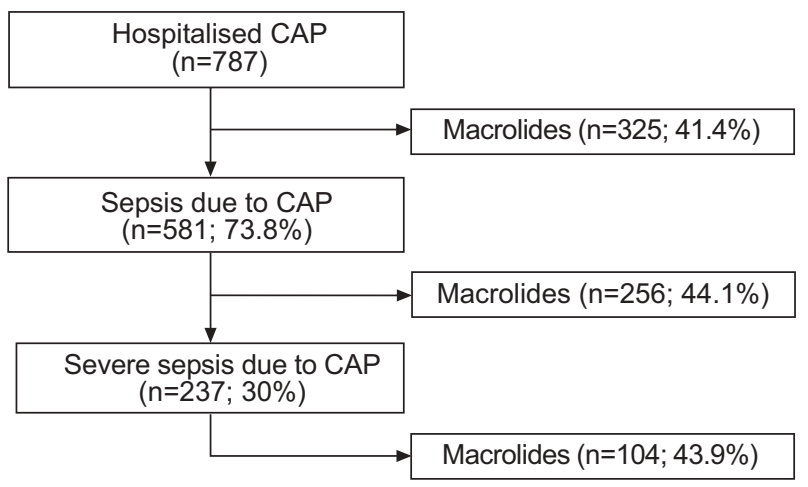

FIGURE 1. Flow diagram of patients admitted to hospital with communityacquired pneumonia (CAP) who developed severe sepsis that required macrolide therapy.

\section{RESULTS}

Initially, 787 CAP patients were identified, out of whom 237 met criteria for severe sepsis (fig. 1) and were included in the analysis. Out of those included, 104 (43.9\%) subjects received a macrolide during the first $48 \mathrm{~h}$ of admission, while 133 did not receive macrolide therapy.

\section{Patient characteristics and microbiological diagnosis}

Table 1 shows the characteristics of the subjects, grouped by whether they received macrolide therapy. There were no significant differences in mean age, sex or pre-existing comorbid conditions, except that cerebrovascular disease $(23 \%$ versus $8 \% ; p=0.002)$ and chronic renal disease $(26 \%$ versus $13 \%$; $p=0.02$ ) were more frequent in subjects who did not receive macrolide therapy.

Physical examination, laboratory and radiological data showed that macrolide-treated subjects were significantly more likely to have hypoxaemia and hyponatraemia (table 1). In general, both groups of subjects had similar severity of illness scores, based on mean \pm SD PSI score $(117 \pm 39$ in non-macrolide subjects compared with $108 \pm 32$ in macrolide treated subjects, $\mathrm{p}=0.2)$.

An aetiological diagnosis was found in 77 (24\%) subjects, more commonly in the macrolide treated group (44 out of $104 ; 42 \%$ ) than in the non-macrolide group (33 out of $133 ; 24.5 \%$ )

TABLE 1 Comparison of demographic and clinical characteristics among severe sepsis patients with community-acquired pneumonia who received initial macrolide versus non-macrolide therapy

\begin{tabular}{|c|c|c|c|}
\hline Variable & Non-macrolide & Macrolide & p-value \\
\hline Patients n & 133 & 104 & \\
\hline Age yrs & $63.3 \pm 17.1$ & $58.8 \pm 15.8$ & 0.2 \\
\hline Male & $109(82.0)$ & $80(76.9)$ & 0.3 \\
\hline \multicolumn{4}{|l|}{ Pre-existing comorbid conditions } \\
\hline Congestive heart failure & $34(25.6)$ & $19(18.3)$ & 0.2 \\
\hline History of stroke & $30(22.6)$ & $8(7.7)$ & 0.002 \\
\hline Chronic liver disease & $17(12.8)$ & $11(10.6)$ & 0.6 \\
\hline History of malignancy & $18(13.5)$ & $7(6.5)$ & 0.09 \\
\hline Renal insufficiency & $34(25.6)$ & $14(13.5)$ & 0.02 \\
\hline Chronic obstructive lung disease & 39 (29.3) & $22(21.2)$ & 0.2 \\
\hline \multicolumn{4}{|c|}{ History, physical, laboratory and radiographical data } \\
\hline Altered mental status & $36(27.1)$ & $32(30.8)$ & 0.5 \\
\hline Respiratory rate $>30 \mathrm{~min}^{-1}$ & $19(14.3)$ & $14(13.5)$ & 0.2 \\
\hline Systolic blood pressure $<90 \mathrm{mmHg}$ & $8(6.0)$ & $8(7.7)$ & 0.6 \\
\hline Cardiac frequency $>125 \mathrm{~min}^{-1}$ & $25(18.8)$ & $19(18.3)$ & 0.9 \\
\hline Temperature $<95^{\circ} \mathrm{F}$ or $>104^{\circ} \mathrm{F}$ & $3(2.3)$ & $3(2.9)$ & 0.8 \\
\hline Arterial $\mathrm{pH}<7.35$ & $19(14.3)$ & $18(17.3)$ & 0.5 \\
\hline $\mathrm{Pa}, \mathrm{O}_{2}<90 \%$ & $39(29.3)$ & $42(40.4)$ & 0.07 \\
\hline $\mathrm{Pa}, \mathrm{O}_{2} / \mathrm{Fi}, \mathrm{O}_{2}$ ratio $\leqslant 250$ & $51(38.4)$ & $60(57.7)$ & 0.07 \\
\hline Haematocrit $<30 \%$ & $12(9.0)$ & $14(13.5)$ & 0.3 \\
\hline Serum blood urea nitrogen $>30 \mathrm{mg} \cdot \mathrm{dL}^{-1}$ & $75(56.4)$ & $49(47.1)$ & 0.2 \\
\hline Serum glucose $>250 \mathrm{mg} \cdot \mathrm{dL}^{-1}$ & $20(15.0)$ & $13(12.5)$ & 0.6 \\
\hline Serum sodium $<130 \mathrm{mEq} \cdot \mathrm{L}^{-1}$ & $18(13.5)$ & $26(25.0)$ & 0.02 \\
\hline Pleural effusion & $36(27.1)$ & $30(28.8)$ & 0.8 \\
\hline Multilobar infiltrates & $58(43.9)$ & $55(52.9)$ & 0.2 \\
\hline \multicolumn{4}{|l|}{ Processes of care measures } \\
\hline Appropriate blood cultures & $108(81.20)$ & $86(82.7)$ & 0.8 \\
\hline Guideline-concordant antibiotic therapy & $84(63.2)$ & $101(97.1)$ & $<0.001$ \\
\hline Antibiotic administration within $4 \mathrm{~h}$ & $39(29.3)$ & $37(35.6)$ & 0.3 \\
\hline
\end{tabular}

Data are presented as mean $\pm \mathrm{SD}$ or $\mathrm{n}(\%)$, unless otherwise stated. $\mathrm{Pa}, \mathrm{O}_{2}$ : arterial oxygen tension; Fi, $\mathrm{O}_{2}$ : inspiratory oxygen fraction 


\begin{tabular}{|c|c|c|c|}
\hline \multirow[t]{2}{*}{ TABLE 2} & \multicolumn{3}{|c|}{$\begin{array}{l}\text { Aetiological diagnosis with an identifiable } \\
\text { pathogen causing disease among severe sepsis } \\
\text { patients with community-acquired pneumonia } \\
\text { who received initial macrolide versus non- } \\
\text { macrolide therapy }\end{array}$} \\
\hline & & Non-macrolide & Macrolide \\
\hline \multicolumn{2}{|l|}{ Patients $\mathrm{n}$} & 133 & 104 \\
\hline \multicolumn{4}{|c|}{ Microorganisms } \\
\hline \multicolumn{2}{|c|}{ Streptococcus pneumoniae } & $13(9.7)$ & $18(17.3)$ \\
\hline \multicolumn{2}{|c|}{ Macrolide-resistant S. pneumoniae } & $5(38.5)$ & $8(44.4)$ \\
\hline \multicolumn{2}{|c|}{ Staphylococcus aureus } & $12(9.0)$ & $10(9.6)$ \\
\hline \multicolumn{2}{|c|}{ Pseudomonas aeruginosa } & $3(2.2)$ & $5(4.8)$ \\
\hline \multicolumn{2}{|c|}{ Haemophilus influenzae } & $1(0.7)$ & $1(1.0)$ \\
\hline \multicolumn{2}{|c|}{ Escherichia coli } & $0(0)$ & $1(1.0)$ \\
\hline \multicolumn{2}{|c|}{ Klebsiella pneumoniae } & $1(0.7)$ & $2(4.5)$ \\
\hline \multicolumn{2}{|c|}{ Proteus mirabilis } & $0(0)$ & $1(1.0)$ \\
\hline \multicolumn{2}{|c|}{ Miscellaneous ${ }^{\#}$} & $1(0.7)$ & $4(3.8)$ \\
\hline \multicolumn{2}{|c|}{ Polymicrobial } & $2(1.5)$ & $2(1.9)$ \\
\hline \multicolumn{2}{|c|}{ Total pathogens isolated } & $33(24.5)$ & $44(42.3)$ \\
\hline \multicolumn{2}{|c|}{ No pathogen isolated } & $100(75.5)$ & $60(57.7)$ \\
\hline
\end{tabular}

Data are presented as $n(\%)$, unless otherwise stated. Percentages have been rounded and may not sum to $100 .{ }^{*}$ : Miscellaneous comprises Acinetobacter spp., Aspergillus spp., Haemophilus parainfluenzae and Streptococcus spp.

(table 2). Of all the CAP subjects in whom a definitive diagnosis was reached, the most frequent pathogen isolated was Streptococcus pneumoniae (13\%), followed by S. aureus (9\%). Macrolide-resistant pathogens were identified in $29 \%$ of the patients with an identifiable microorganism (table 3).

\section{Clinical outcomes}

Overall, 30- and 90-day mortality was lower for subjects who received macrolides compared with non-macrolide subjects
( $11 \%$ versus $29 \% ; p=0.001$, and $12 \%$ versus $34 \% ; p<0.001$, respectively; fig. 2). Mean $\pm \mathrm{SD}$ length of hospital stay was similar in non-macrolide subjects (9.2 \pm 9.5 days) compared with macrolide patients $(10.6 \pm 10.1$ days; $\mathrm{p}=0.5)$. Both groups had similar rates of ICU admission ( $49 \%$ versus $44 \%$; $=0.4$ ), need for mechanical ventilation $(18 \%$ versus $16 \% ; \mathrm{p}=0.3)$, and need for vasopressors ( $7 \%$ versus $11 \% ; \mathrm{p}=0.3)$. In the subgroup analysis, macrolide-treated subjects with severe sepsis were more likely to survive at 30 and 90 days (table 3 ) if the cultures were negative $(p=0.005)$, the cultures were positive $(p=0.004)$, or they were infected with Gram-negative rods $(p=0.002)$, and for all the macrolide-resistant pathogens, compared with nonmacrolide subjects.

In the Cox proportional hazard model, after adjusting for potential confounders, including the propensity score, those subjects who received macrolide therapy had a lower risk of dying within 30 days (hazard ratio (HR) 0.3, 95\% confidence interval (CI) $0.2-0.7$ ) and 90 days (HR 0.3, 95\% CI 0.2-0.6) of admission (fig. 2). This survival benefit remained after evaluating patients with severe sepsis with culture-negative results, culture-positive data and macrolide-resistant pathogens (fig. 2).

\section{DISCUSSION}

The present authors found that subjects with severe sepsis due to CAP had improved survival at 30 and 90 days when they received empirical macrolide therapy at the time of hospital admission. This survival benefit remained after evaluating all the cases with documented macrolide-resistant pathogens and severe sepsis. The patients studied included the more severe end of the spectrum of CAP. Only CAP patients admitted to the hospital directly from home with severe sepsis, defined by the presence of at least one organ dysfunction, were included [23]. ICU admission occurred in almost half of both study groups, with $\sim 20 \%$ receiving mechanical ventilation. A number of comorbid conditions (COPD, congestive heart failure, chronic renal disease and cerebrovascular disease) were present,

\begin{tabular}{|c|c|c|c|}
\hline \multirow[t]{2}{*}{ Patient cohorts } & \multicolumn{3}{|c|}{ Mortality at 90 days } \\
\hline & Non-macrolide & Macrolide & p-value \\
\hline Severe sepsis ${ }^{\#}$ & 45/133 (33.8) & 13/104 (12.5) & $<0.0001$ \\
\hline Streptococcus pneumoniae & $5 / 13(38.4)$ & $4 / 18(22.2)$ & 0.3 \\
\hline Macrolide susceptible S. pneumoniae & $3 / 7(42.9)$ & $3 / 7(42.9)$ & 1.0 \\
\hline Macrolide resistant S. pneumoniae & $2 / 5(40.0)$ & $1 / 8(12.5)$ & 0.3 \\
\hline Methicillin-resistant Staphylococcus aureus & $2 / 5(40.0)$ & $0 / 2(0)$ & 0.3 \\
\hline Gram-negative rods & $3 / 4(75.0)$ & $0 / 10(0)$ & 0.002 \\
\hline
\end{tabular}

Data are presented as $\mathrm{n} / \mathrm{N}(\%)$. Percentages have been rounded and may not sum to 100. ${ }^{*}$ : mortality at 30 days in the macrolide and non-macrolide groups was similar for most of the cohorts except for: severe sepsis (37 out of 133 (27.8\%) versus 11 out of 104 (10.6\%); $p=0.001$ ); culture-negative sepsis (24 out of 100 (24.0\%) versus six out of 60 (10.0\%); $p=0.03$ ); and culture-positive sepsis (13 out of 33 (39.4\%) versus five out of $44(11.4 \%) ; p=0.004)$. $\because$ : Gram-negative rods include Pseudomonas aeruginosa, Escherichia coli, Klebsiella pneumoniae, Proteus mirabilis and Acinetobacter spp. 

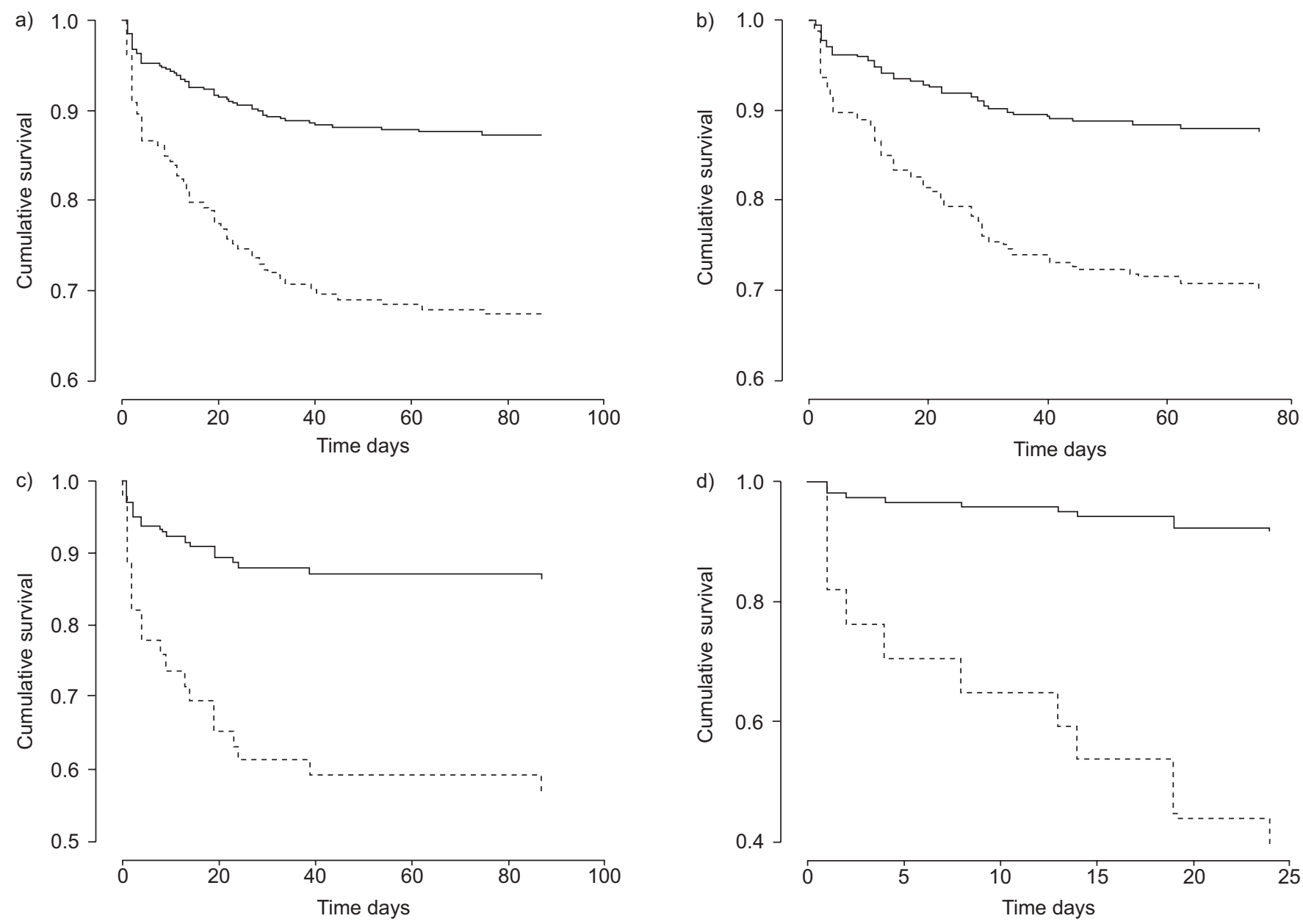

FIGURE 2. Cox survival curves of patients with severe sepsis due to community-acquired pneumonia who received initial macrolide versus non-macrolide therapy. a) All patients (hazard ratio (HR) $0.34,95 \%$ confidence interval $(\mathrm{Cl}) 0.18-0.66$; $\mathrm{p}=0.001)$; b) patients with culture-negative severe sepsis $(\mathrm{HR} 0.37,95 \% \mathrm{Cl} 0.16-0.87 ; \mathrm{p}=0.02)$; $\mathrm{c}$ ) patients with culture-positive severe sepsis ( $\mathrm{HR} 0.26,95 \% \mathrm{Cl} 0.09-0.73 ; \mathrm{p}=0.01)$; d) patients with macrolide-resistant severe sepsis $(\mathrm{HR} 0.10,95 \% \mathrm{Cl} 0.02-0.49 ; \mathrm{p}=0.005)$. - . - - no macrolides; __ : macrolides.

whether or not initial macrolide therapy was used. A mortality rate of almost $30 \%$ in the group of patients who did not receive macrolide therapy is similar to that reported in the placebo group of subjects with severe sepsis enrolled in the Recombinant Human Activated Protein C Worldwide Evaluation in Severe Sepsis (PROWESS) trial [3]. However, some differences in baseline characteristics were observed in the macrolide therapy group versus non-macrolide patients. Patients receiving macrolides were less likely to have a history of cerebrovascular disease and chronic renal disease, despite no statistical differences in the PSI score. All of these clinical differences are accounted for in the PSI score, which was the instrument to adjust for severity of illness in the current study [28]. The magnitude of the benefit is important compared with prior reports of other therapies used in patients with severe sepsis. The observed benefit of macrolide therapy was not explained by differences in guideline-concordant therapy between groups. The fact that almost all patients with severe sepsis due to CAP received guideline-concordant therapy in the present study suggests that the observed reduction in mortality may be driven by the use of a macrolide in hospitalised patients (whether on the ward or in the ICU). Interestingly, the survival curves for patients with severe sepsis due to CAP separated within the first few days after receiving macrolide therapy, similar to patterns seen with other immunomodulatory agents [3]. The benefit observed in short-term (30-day) mortality is also demonstrated with longterm (90-day) mortality.

The suggested benefit of macrolide therapy in patients with respiratory infections is demonstrated by clinical studies of patients with pneumococcal pneumonia and CAP. WATERER et al. [14], demonstrated that the use of a single effective antibiotic against pneumococcus was associated with an increased mortality compared with the use of two effective agents. Macrolide-containing combinations were the most common type of combination therapy and had lower mortality than predicted by the Acute Physiology and Chronic Health Evaluation (APACHE) II prediction model. Several other studies have suggested a benefit of having a macrolide added to $\beta$-lactam therapy in patients with bacteraemic pneumococcal pneumonia [11-16]. Not adding a macrolide to a $\beta$-lactam-based 
initial antibiotic regimen was an independent predictor of inhospital mortality [12]. A recent study by RODRIGUEZ et al. [20] showed in a multicentre, prospective cohort in ICU-admitted CAP patients with septic shock that 28-day ICU survival was higher with combination therapy in CAP patients with shock (HR 1.69, 95\% CI 1.09-2.60; $\mathrm{p}=0.01$ ) and in patients who received macrolide therapy (HR 1.73, 95\% CI 1.08-2.76; $\mathrm{p}=0.02$ ). The majority of combination therapies in these studies included a macrolide. These results suggest that the benefit of macrolides may not be limited to bacteraemic pneumococcal disease or even to pneumococcal CAP. However, a recent randomised controlled trial in patients with sepsis due to ventilator-associated pneumonia treated with clarithromycin did not show a survival benefit [29]. However, clarithromycin accelerated the resolution of ventilator-associated pneumonia, and weaning from mechanical ventilation in surviving patients and delayed death in those who died of sepsis.

The reasons why macrolides may contribute to better outcomes in patients with severe sepsis due to CAP include: 1) a synergistic antibacterial mechanism; 2) atypical pathogen coverage; and 3) an immunomodulatory effect. Synergism is unlikely to be the reason, because Gram-positive pathogens are not the only cause of severe sepsis due to CAP. Almost 30\% of the positive cultures were bacteria other than Gram-positive microorganisms [1]. Atypical coverage is also unlikely, since the present authors have reported previously that combination therapy with a fluoroquinolone had no survival advantage [30]. The most likely explanation of this effect is the immunomodulation produced by the macrolide antibiotic affecting the cytokine response and inflammatory response at different levels. The present authors strongly suggest that immunomodulation is likely to have occurred, and the current data support the benefit of macrolide therapy even in those patients with documented negative cultures, Gram-negative bacilli and all macrolide-resistant pathogens.

Several publications have supported the benefit of macrolides not only for their antimicrobial properties but for their effect on immune function. Some examples of this effect include noninfectious conditions including asthma [8], COPD [9], diffuse panbronchiolitis [10] and bronchiectasis [10]. The most dramatic example of the efficacy of macrolides as immune modulators is in diffuse panbronchiolitis. This noninfectious, autoimmune lung condition had a 70\% 5-yr mortality rate until long-term low-dose erythromycin was found to reduce 5-yr mortality to $<20 \%$ [10].

The current study has limitations that are important to acknowledge. First, it was a retrospective cohort study, and there are inherent problems related to this design, including selection bias. However, the current authors do not feel that the study has significant problems with either bias, due to the use of admission and discharge diagnosis ICD-9 codes to identify patients and the fact that only a small amount of missing data was encountered. Moreover, it was possible to verify that all the patients had a radiological diagnosis of CAP. It is important to recognise that the APACHE II score could not be calculated due to some missing variables, and one of the limitations of the PSI data collection in retrospective studies is the assessment of missing or undocumented data as "normal". The second limitation of the present study is the post hoc definition and analysis of the severe sepsis subgroup. However, it is well known that CAP is an important cause of severe sepsis [1-3] and treatment strategies in patients with severe sepsis depend on the site of infection. The current data was limited regarding documentation of other interventions that are currently used in patients with severe sepsis or septic shock, such as corticosteroids and activated protein C. However, the studies that suggested these interventions were published at the end of the study period and due to slow implementation in the two participating hospitals, this may not have altered the results of the macrolide study. Thirdly, the current sample was predominantly male, since one of the sites was a Veterans Affairs Medical Center. The current authors are unable to examine whether female CAP patients admitted to the hospital may have a different clinical course, or outcomes, as compared with males.

The present results support the current recommendations about the addition of macrolide therapy in ward patients with CAP and also in ICU patients. In addition, the data suggests that providing appropriate guideline-concordant therapy alone may not be enough to change mortality significantly. The addition of macrolides may have benefits for severely ill patients other than just antibiotic coverage. The present authors hypothesise that a benefit related to immunomodulation may be as important as, or more important than, the antibiotic effect, and might be useful in the treatment of patients with severe sepsis due to other infectious conditions.

In conclusion, the present study demonstrates that macrolide use in subjects with severe sepsis was associated with decreased mortality at both 30 and 90 days, even in those cases in which macrolide resistance was documented. The decreased mortality associated with macrolide therapy was consistent despite the adjustment of other variables previously described as being associated with mortality. Further prospective studies are needed to confirm these findings, as well as to determine whether treatment with a macrolide might be beneficial for patients with severe sepsis secondary to other infections.

\section{REFERENCES}

1 Martin GS, Mannino DM, Eaton S, Moss M. The epidemiology of sepsis in the United States from 1979 through 2000. N Engl J Med 2003; 348: 1546-1554.

2 Angus DC, Linde-Zwirble WT, Lidicker J, Clermont G, Carcillo J, Pinsky MR. Epidemiology of severe sepsis in the United States: analysis of incidence, outcome, and associated costs of care. Crit Care Med 2001; 29: 1303-1310.

3 Bernard GR, Vincent JL, Laterre PF, et al. Efficacy and safety of recombinant human activated protein $\mathrm{C}$ for severe sepsis. N Engl J Med 2001; 344: 699-709.

4 Hoyert DL, Kung HC, Smith BL. Deaths: preliminary data for 2003. Natl Vital Stat Rep 2005; 53: 1-48.

5 Gilbert K, Fine MJ. Assessing prognosis and predicting patient outcomes in community-acquired pneumonia. Semin Respir Infect 1994; 9: 140-152.

6 Dellinger RP, Levy MM, Carlet JM, et al. Surviving Sepsis Campaign: international guidelines for management of severe sepsis and septic shock: 2008. Crit Care Med 2008; 36: 296-327. 
7 Giamarellos-Bourboulis EJ, Adamis T, Laoutaris G, et al. Immunomodulatory clarithromycin treatment of experimental sepsis and acute pyelonephritis caused by multidrug-resistant Pseudomonas aeruginosa. Antimicrob Agents Chemother 2004; 48: 93-99.

8 Amsden GW. Anti-inflammatory effects of macrolides - an underappreciated benefit in the treatment of communityacquired respiratory tract infections and chronic inflammatory pulmonary conditions? J Antimicrob Chemother 2005; 55: 10-21.

9 Parnham MJ, Culic O, Erakovic V, et al. Modulation of neutrophil and inflammation markers in chronic obstructive pulmonary disease by short-term azithromycin treatment. Eur J Pharmacol 2005; 517: 132-143.

10 Schultz MJ. Macrolide activities beyond their antimicrobial effects: macrolides in diffuse panbronchiolitis and cystic fibrosis. J Antimicrob Chemother 2004; 54: 21-28.

11 Baddour LM, Yu VL, Klugman KP, et al. Combination antibiotic therapy lowers mortality among severely ill patients with pneumococcal bacteremia. Am J Respir Crit Care Med 2004; 170: 440-444.

12 Martinez JA, Horcajada JP, Almela M, et al. Addition of a macrolide to a $\beta$-lactam-based empirical antibiotic regimen is associated with lower in-hospital mortality for patients with bacteremic pneumococcal pneumonia. Clin Infect Dis 2003; 36: 389-395.

13 Metersky ML, Ma A, Houck PM, Bratzler DW. Antibiotics for bacteremic pneumonia: improved outcomes with macrolides but not fluoroquinolones. Chest 2007; 131: 466-473.

14 Waterer GW, Somes GW, Wunderink RG. Monotherapy may be suboptimal for severe bacteremic pneumococcal pneumonia. Arch Intern Med 2001; 161: 1837-1842.

15 Weiss K, Low DE, Cortes L, et al. Clinical characteristics at initial presentation and impact of dual therapy on the outcome of bacteremic Streptococcus pneumoniae pneumonia in adults. Can Respir J 2004; 11: 589-593.

16 Lujan M, Gallego M, Fontanals D, Mariscal D, Rello J. Prospective observational study of bacteremic pneumococcal pneumonia: effect of discordant therapy on mortality. Crit Care Med 2004; 32: 625-631.

17 Garcia Vazquez E, Mensa J, Martinez JA, et al. Lower mortality among patients with community-acquired pneumonia treated with a macrolide plus a $\beta$-lactam agent versus a $\beta$-lactam agent alone. Eur J Clin Microbiol Infect Dis 2005; 24: 190-195.

18 Houck PM, MacLehose RF, Niederman MS, Lowery JK. Empiric antibiotic therapy and mortality among medicare pneumonia inpatients in 10 western states: 1993, 1995, and 1997. Chest 2001; 119: 1420-1426.

19 Mortensen EM, Restrepo M, Anzueto A, Pugh J. Effects of guideline-concordant antimicrobial therapy on mortality among patients with community-acquired pneumonia. Am J Med 2004; 117: 726-731.

20 Rodriguez A, Mendia A, Sirvent JM, et al. Combination antibiotic therapy improves survival in patients with community-acquired pneumonia and shock. Crit Care Med 2007; 35: 1493-1498.

21 Cazzola M, Saltini C. Novel approaches to the treatment of bacterial lower respiratory tract infections: introduction. Respiration 2005; 72: 7-8.

22 Parnham MJ. Immunomodulatory effects of antimicrobials in the therapy of respiratory tract infections. Curr Opin Infect Dis 2005; 18: 125-131.

23 Bone RC. Why sepsis trials fail. JAMA 1996; 276: 565-566.

24 Mortensen EM, Coley CM, Singer DE, et al. Causes of death for patients with community-acquired pneumonia: results from the Pneumonia Patient Outcomes Research Team cohort study. Arch Intern Med 2002; 162: 1059-1064.

25 Mandell LA, Wunderink RG, Anzueto A, et al. Infectious Diseases Society of America/American Thoracic Society Consensus guidelines on the management of communityacquired pneumonia in adults. Clin Infect Dis 2007; 44: Suppl. 2, S27-S72.

26 National Committee for Clinical Laboratory Standards. Performance Standards for Antimicrobial Susceptibility Testing. 11th Informational Supplement. Wayne, National Committee for Clinical Laboratory Standards, 2001.

27 Stone RA, Obrosky DS, Singer DE, Kapoor WN, Fine MJ. Propensity score adjustment for pretreatment differences between hospitalized and ambulatory patients with community-acquired pneumonia. Pneumonia Patient Outcomes Research Team (PORT) Investigators. Med Care 1995; 33: Suppl. 4, AS56-AS66.

28 Fine MJ, Auble TE, Yealy DM, et al. A prediction rule to identify low-risk patients with community-acquired pneumonia. N Engl J Med 1997; 336: 243-250.

29 Giamarellos-Bourboulis EJ, Pechere JC, Routsi C, et al. Effect of clarithromycin in patients with sepsis and ventilator associated pneumonia. Clin Infect Dis 2008; 46 1157-1164.

30 Mortensen EM, Restrepo MI, Anzueto A, Pugh J. The impact of empiric antimicrobial therapy with a $\beta$-lactam and fluoroquinolone on mortality for patients hospitalized with severe pneumonia. Crit Care 2006; 10: R8. 